\title{
Evaluation of detection efficiency on radar network
}

\author{
Yujuan Cui ${ }^{1, a}$, Hao Cha ${ }^{2, b}$ \\ ${ }^{1}$ Naval University of Engineering, Wuhan, Hubei, China,430033 \\ ${ }^{2}$ Naval University of Engineering, Wuhan,Hubei, China,430033 \\ aemail:xiaoxiao926878@126.com, bemail:310938289@qq.com
}

Keywords: radar network; detection efficiency; analytic hierarchy process (AHP); consistency

\begin{abstract}
In order to evaluation on efficiency of radar network detection, the evaluation index system on efficiency of radar network detection was established on the basis of construction principles. Considering the key and difficult in analytic hierarchy process, judgment matrix which satisfies the consistency test is proposed. Finally, the rationalization of the model is validated by use of this way which avoids lots of computations and deals with difficulty of analytic hierarchy process.
\end{abstract}

\section{Introduction}

In view of the meaning of network, radar network system realizes information sharing, detect and scout the aerial target through mutual complementarity the different programs, system, frequency radars, effectively enhances the detection ability, in the meantime, the gained incoming target information can be effective early warning and guidance, improve the ability to survive. References [1-6] respectively study the performance of the radar network in four aspects of anti-interference performance, anti-stealth performance, destroy resistance and low altitude detection performance, which consolidate the basis of detecting efficiency evaluation. References [7-8] make use of AHP and SAE to evaluate detecting efficiency.

The difficulty of AHP is judgment matrix. On the one hand, judgment matrix can objectively reflect the actual importance degree, on the other hand, judgment matrix has good consistency. But in the actual judgment matrix, its consistency test is not passed and the more matrix order, the more complexity of the consistency check. To this end, this paper proposes a new method to construct judgment matrix with completely consistent and the improved AHP is applied to radar network, solves the problem of effectiveness evaluation, so as to improve the practicability and reliability of the AHP.

\section{Evaluation index system for radar network detection efficiency}

Construction principle. The detection performance indicators should be selected in line with the overall integrity, reasonable scientific, independent consistency and simple principle. To true, comprehensively and objectively reflect radar network detection efficiency. The five main factors are analyzed from the detection coverage, detection accuracy, detection and control ability , anti-jamming capability.

Index system. Based on the above principle and study of related radar data, the evaluation index system with 5 main factors and 27 sub-factors is set up. Specific content can be shown in the figure 1 


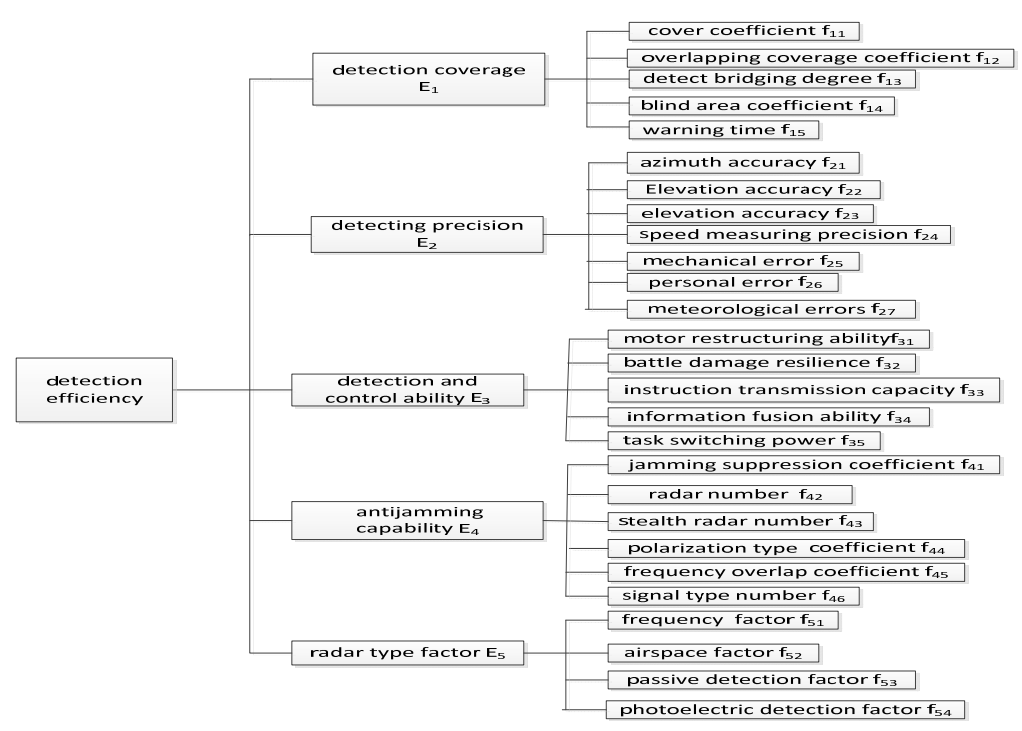

Fig.1 The composition of index system

\section{Improved AHP evaluates index system}

The analytic hierarchy process ${ }^{[9]}$ is firstly proposed since the 1970 s, and now has got things right on a number of fronts in areas such as society, economy, management.so it has been attention widely in the world. Given that it is a combination of qualitative and quantitative decision method, some obvious actual defects are exposed in the application of specific practical problems, such as judgment matrix often does not satisfy consistency. But the following problems, such as large amount of calculation, complex calculation process and relatively large errors, make scholars miserable.

Theorem 1 Assuming that $A=\left(\mathbf{a}_{i j}\right)_{n \times n}$ is n-order matrix as shown in the equation (1), here $\boldsymbol{q}_{1}, \boldsymbol{q}_{2}, \cdots, \boldsymbol{q}_{n-1}$ are positive real number.

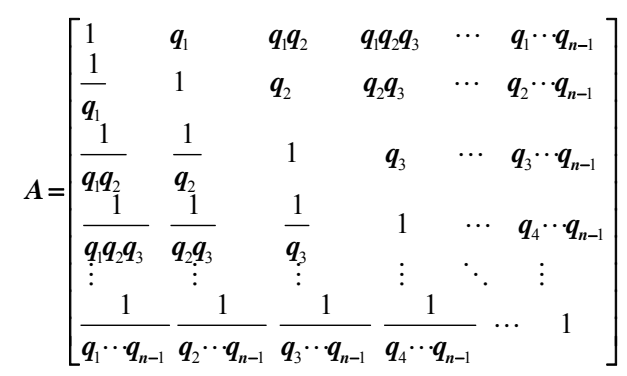

Then matrix $\boldsymbol{A}$ is absolute consistency and the corresponding eigenvector $\boldsymbol{X}$ of maximal eigenvalue $n$ is as follows. The normalized eigenvector $\bar{X}$ is also obtained.

$$
\begin{aligned}
& \boldsymbol{X}=\left(\boldsymbol{q}_{1} \boldsymbol{q}_{2} \cdots \boldsymbol{q}_{n-1}, \boldsymbol{q}_{2} \boldsymbol{q}_{3} \cdots \boldsymbol{q}_{n-1}, \cdots, \boldsymbol{q}_{n-1}, 1\right)^{T} \\
& \overline{\boldsymbol{X}}=\left(\frac{\boldsymbol{q}_{1} \boldsymbol{q}_{2} \cdots \boldsymbol{q}_{n-1}}{\boldsymbol{Y}}, \frac{\boldsymbol{q}_{2} \boldsymbol{q}_{3} \cdots \boldsymbol{q}_{n-1}}{\boldsymbol{Y}}, \cdots, \frac{\boldsymbol{q}_{n-1}}{\boldsymbol{Y}}, \frac{1}{\boldsymbol{Y}}\right)^{T} \\
& \boldsymbol{Y}=\boldsymbol{q}_{1} \boldsymbol{q}_{2} \cdots \boldsymbol{q}_{n-1}+\boldsymbol{q}_{2} \boldsymbol{q}_{3} \cdots \boldsymbol{q}_{n-1},+\cdots+\boldsymbol{q}_{n-1}+1
\end{aligned}
$$

The prove process is omitted ${ }^{[10]}$,

Assuming that the overall objective $\boldsymbol{D}$ includes $\boldsymbol{m}$ indexes $\boldsymbol{E}_{1}, \boldsymbol{E}_{2}, \cdots, \boldsymbol{E}_{\boldsymbol{m}}$, which respectively have weight values $\boldsymbol{e}_{1}, \boldsymbol{e}_{2}, \cdots, \boldsymbol{e}_{\boldsymbol{m}}$; There are $\boldsymbol{n}$ sub-indexes $\boldsymbol{F}_{j}$, the weight values of $\boldsymbol{F}_{j}$ relative to $\boldsymbol{E}_{j}(\boldsymbol{j}=1,2, \cdots, \boldsymbol{m})$ are $\boldsymbol{f}_{1 j}, \boldsymbol{f}_{2 j}, \cdots, \boldsymbol{f}_{n j}$, if there are no relations between $\boldsymbol{F}_{j}$ and $\boldsymbol{E}_{i}$, then $\boldsymbol{f}_{j i}=0$. So the synthetic level weight values of sub-indexes can make use of the equation (5) to calculate.

$$
\omega_{i j}=\sum_{k=1}^{m} \boldsymbol{e}_{i} \boldsymbol{f}_{j k}
$$

On the base of mentioned above ,the evaluation process of radar network detecting efficiency can be divided into the following five steps:

Step1 Establish evaluation set. Here evaluation set $v=\left\{v_{1}, v_{2}, \cdots, v_{5}\right\}=\{$ equally important, somewhat important, obviously important, very important, absolutely important $\}$. 
Step2 Construct the judgment matrices. According to the equation (1) to construct judgment matrix, attention should be paid to the objectivity of importance scale between the factors.

Step 3 Determine the priorities of factors in all levels. According to the equation (2),equation(3) and equation(4), to construct judgment matrix, the normalized eigenvectors are got, which are the sequencing weight values of relative importance of various factors relative to the target.

Step 4 Determine the total sequencing weight vector of each level's indexes.By the equation (5), the total sequencing weight vector can be obtained.

Step 5 Compute efficiency evaluation.According to the equation (6), the sum of all indicators effectiveness value is the total effectiveness evaluation.

$$
W=\sum_{i} \sum_{j} \omega_{i j} x_{i j}
$$

\section{Simulation and Analysis}

In this section, we present an example to illustrate radar network detection of the proposed algorithms. Table 1 is the actual and optimal values of detecting efficiency indexes. Now, analyzing the detecting efficiency of radar network is as followed. By analyzing amount of data and expert scoring method, judgment matrix of five main factors is obtained in table 2.It is easy to get maximum eigenvalue and its corresponding eigenvector $\overline{x_{0}}$ by the above way.$\overline{x_{0}}$ which is the result after normalization of $\overline{x_{0}}$, is single hierarchical arrangement.

$$
\begin{gathered}
\boldsymbol{x}_{0}=(3.7,1.2333,1.85,1.5417,1)^{T} \\
\overline{\mathbf{x}_{0}}=(0.3968,0.1323,0.1984,0.1653,0.1072)^{T}
\end{gathered}
$$

In the same manner, from table 3 to table 7, judgment matrixes of $E_{i}-f_{i j}$ are given. And then the corresponding eigenvectors of their maximum eigenvalues are single hierarchical arrangement as showed following.

$$
\begin{aligned}
& \overline{\mathbf{x}_{1}}=(0.2591,0.1178,0.0617,0.0432,0.5182)^{T}, \overline{\mathbf{x}_{2}}=(0.3692,0.1477,0.2172,0.0820,0.0568,0.0820,0.0451)^{T} \\
& \overline{\mathbf{x}_{3}}=(0.1496,0.0427,0.2992,0.4487,0.0598)^{T}, \overline{\mathbf{x}_{4}}=(0.4094,0.1637,0.0975,0.0682,0.1637,0.0975)^{T} \\
& \overline{\mathbf{x}_{5}}=(0.1351,0.5405,0.2703,0.0541)^{T}
\end{aligned}
$$

Tab 1 The actual and optimal scoring table of indicators

\begin{tabular}{cccccccc}
$j \mathrm{j}$ & 1 & 2 & 3 & 4 & 5 & 6 & 7 \\
\hline 1 & $8(9)$ & $6(7)$ & $7(9)$ & $7(9)$ & $7(8)$ & & \\
2 & $5(8)$ & $5(8)$ & $6(8)$ & $5(8)$ & $5(7)$ & $5(7)$ & $5(7)$ \\
3 & $7(9)$ & $6(8)$ & $8(9)$ & $7(9)$ & $6(7)$ & & \\
4 & $6(7)$ & $6(7)$ & $3(5)$ & $8(9)$ & $6(8)$ & $6(7)$ & \\
5 & $5(7)$ & $5(7)$ & $6(7)$ & $5(8)$ & & & \\
\hline
\end{tabular}

(1) Number in bracket is optimal value of this index, number outside bracket is the corresponding actual value Tab 2 The judgment matrix $\boldsymbol{D}-\boldsymbol{E}_{\boldsymbol{i}}$

\begin{tabular}{cccccc}
\hline $\boldsymbol{D}-\boldsymbol{E}_{\boldsymbol{i}}$ & $\boldsymbol{E}_{1}$ & $\boldsymbol{E}_{2}$ & $\boldsymbol{E}_{3}$ & $\boldsymbol{E}_{4}$ & $\boldsymbol{E}_{5}$ \\
\hline $\boldsymbol{E}_{1}$ & 1 & 3 & 2 & 2.4 & 3.7 \\
$\boldsymbol{E}_{2}$ & $1 / 3$ & 1 & $2 / 3$ & $2.4 / 3$ & $3.7 / 3$ \\
$\boldsymbol{E}_{3}$ & $1 / 2$ & $3 / 2$ & 1 & $2.4 / 2$ & $3.7 / 2$ \\
$\boldsymbol{E}_{4}$ & $1 / 2.4$ & $3 / 2.4$ & $2 / 2.4$ & 1 & $3.7 / 2.4$ \\
$\boldsymbol{E}_{5}$ & $1 / 3.7$ & $3 / 3.7$ & $2 / 3.7$ & $2.4 / 3.7$ & 1 \\
\hline
\end{tabular}

Tab 3 The judgment matrix $\boldsymbol{E}_{1}-\boldsymbol{f}_{1 j}$

\begin{tabular}{cccccc}
\hline $\boldsymbol{E}_{1}-\boldsymbol{f}_{1 j}$ & $\boldsymbol{f}_{11}$ & $\boldsymbol{f}_{12}$ & $\boldsymbol{f}_{13}$ & $\boldsymbol{f}_{14}$ & $\boldsymbol{f}_{15}$ \\
\hline $\boldsymbol{f}_{11}$ & 1 & 2.2 & 4.2 & 6 & 0.5 \\
$\boldsymbol{f}_{12}$ & $1 / 2.2$ & 1 & $4.2 / 2.2$ & $6 / 2.2$ & $0.5 / 2.2$ \\
$\boldsymbol{f}_{13}$ & $1 / 4.2$ & $2.2 / 4.2$ & 1 & $6 / 4.2$ & $0.5 / 4.2$ \\
$\boldsymbol{f}_{14}$ & $1 / 6$ & $2.2 / 6$ & $4.2 / 6$ & 1 & $0.5 / 6$ \\
$\boldsymbol{f}_{15}$ & $1 / 0.5$ & $2.2 / 0.5$ & $4.2 / 0.5$ & $6 / 0.5$ & 1 \\
\hline
\end{tabular}


Tab 4 The judgment matrix $\boldsymbol{E}_{2}-\boldsymbol{f}_{2 i}$

\begin{tabular}{cccccccc}
\hline $\boldsymbol{E}_{2}-\boldsymbol{f}_{2 j}$ & $\boldsymbol{f}_{21}$ & $\boldsymbol{f}_{22}$ & $\boldsymbol{f}_{23}$ & $\boldsymbol{f}_{24}$ & $\boldsymbol{f}_{25}$ & $\boldsymbol{f}_{26}$ & $\boldsymbol{f}_{27}$ \\
\hline $\boldsymbol{f}_{21}$ & 1 & 2.5 & 1.7 & 4.5 & 6.5 & 4.5 & 8.2 \\
$\boldsymbol{f}_{22}$ & $1 / 2.5$ & 1 & $1.7 / 2.5$ & $4.5 / 2.5$ & $6.5 / 2.5$ & $4.5 / 2.5$ & $8.2 / 2.5$ \\
$\boldsymbol{f}_{23}$ & $1 / 1.7$ & $2.5 / 1.7$ & 1 & $4.5 / 1.7$ & $6.5 / 1.7$ & $4.5 / 1.7$ & $8.2 / 1.7$ \\
$\boldsymbol{f}_{24}$ & $1 / 4.5$ & $2.5 / 4.5$ & $1.7 / 4.5$ & 1 & $6.5 / 4.5$ & $4.5 / 4.5$ & $8.2 / 4.5$ \\
$\boldsymbol{f}_{25}$ & $1 / 6.5$ & $2.5 / 6.5$ & $1.7 / 6.5$ & $4.5 / 6.5$ & 1 & $4.5 / 6.5$ & $6.5 / 4.5$ \\
$\boldsymbol{f}_{26}$ & $1 / 4.5$ & $2.5 / 4.5$ & $1.7 / 4.5$ & $4.5 / 4.5$ & $6.5 / 4.5$ & 1 & $8.2 / 4.5$ \\
$\boldsymbol{f}_{27}$ & $1 / 8.2$ & $2.5 / 8.2$ & $1.7 / 8.2$ & $4.5 / 8.2$ & $6.5 / 8.2$ & $4.5 / 8.2$ & 1 \\
\hline
\end{tabular}

Tab 5 The judgment matrix $\boldsymbol{E}_{3}-\boldsymbol{f}_{3 j}$

\begin{tabular}{cccccc}
\hline $\boldsymbol{E}_{3}-\boldsymbol{f}_{3 j}$ & $\boldsymbol{f}_{31}$ & $\boldsymbol{f}_{32}$ & $\boldsymbol{f}_{33}$ & $\boldsymbol{f}_{34}$ & $\boldsymbol{f}_{35}$ \\
\hline $\boldsymbol{f}_{31}$ & 1 & 3.5 & 0.5 & $1 / 3$ & 2.5 \\
$\boldsymbol{f}_{32}$ & $1 / 3.5$ & 1 & $0.5 / 3.5$ & $2 / 21$ & $2.5 / 3.5$ \\
$\boldsymbol{f}_{33}$ & $1 / 0.5$ & $3.5 / 0.5$ & 1 & $2 / 3$ & $2.5 / 0.5$ \\
$\boldsymbol{f}_{34}$ & 3 & 10.5 & $3 / 2$ & 1 & $15 / 2$ \\
$\boldsymbol{f}_{35}$ & $1 / 2.5$ & $3.5 / 2.5$ & $0.5 / 2.5$ & $2 / 15$ & 1 \\
\hline
\end{tabular}

Tab 6 The judgment matrix $\boldsymbol{E}_{4}-\boldsymbol{f}_{4 j}$

\begin{tabular}{ccccccc}
\hline $\boldsymbol{E}_{4}-\boldsymbol{f}_{4 j}$ & $\boldsymbol{f}_{41}$ & $\boldsymbol{f}_{42}$ & $\boldsymbol{f}_{43}$ & $\boldsymbol{f}_{44}$ & $\boldsymbol{f}_{45}$ & $\boldsymbol{f}_{46}$ \\
\hline $\boldsymbol{f}_{41}$ & 1 & 2.5 & 4.2 & 6 & 2.5 & 4.2 \\
$\boldsymbol{f}_{42}$ & $1 / 2.5$ & 1 & $4.2 / 2.5$ & $6 / 2.5$ & $2.5 / 2.5$ & $4.2 / 2.5$ \\
$\boldsymbol{f}_{43}$ & $1 / 4.2$ & $2.5 / 4.2$ & 1 & $6 / 4.2$ & $2.5 / 4.2$ & $4.2 / 4.2$ \\
$\boldsymbol{f}_{44}$ & $1 / 6$ & $2.5 / 6$ & $4.2 / 6$ & 1 & $2.5 / 6$ & $4.2 / 6$ \\
$\boldsymbol{f}_{45}$ & $1 / 2.5$ & $2.5 / 2.5$ & $4.2 / 2.5$ & $6 / 2.5$ & 1 & $4.2 / 2.5$ \\
$\boldsymbol{f}_{46}$ & $1 / 4.2$ & $25 / 4.2$ & $4.2 / 4.2$ & $6 / 4.2$ & $2.5 / 4.2$ & 1 \\
\hline
\end{tabular}

Tab 7 The judgment matrix $\boldsymbol{E}_{5}-\boldsymbol{f}_{5 j}$

\begin{tabular}{ccccc}
\hline $\boldsymbol{E}_{5}-\boldsymbol{f}_{5 j}$ & $\boldsymbol{f}_{51}$ & $\boldsymbol{f}_{52}$ & $\boldsymbol{f}_{53}$ & $\boldsymbol{f}_{54}$ \\
\hline $\boldsymbol{f}_{51}$ & 1 & $1 / 4$ & $1 / 2$ & 2.5 \\
$\boldsymbol{f}_{52}$ & 4 & 1 & 2 & 10 \\
$\boldsymbol{f}_{53}$ & 2 & $2 / 4$ & 1 & 5 \\
$\boldsymbol{f}_{54}$ & $1 / 2.5$ & $1 / 10$ & $2 / 10$ & 1
\end{tabular}

According to equation (6),each index can be obtained relative to the level of the overall goal,that is $f_{i j}$ corresponding weighting $\omega_{i j}$, as shown below.

$\omega_{11}=0.1028, \omega_{12}=0.0467, \omega_{13}=0.0245, \omega_{14}=0.0172, \omega_{15}=0.2056, \omega_{21}=0.0488, \omega_{22}=0.0195, \omega_{23}=0.0287, \omega_{24}=0.0109$, $\omega_{25}=0.0075, \omega_{26}=0.0109, \omega_{27}=0.0060 \omega_{31}=0.0297, \omega_{32}=0.0085, \omega_{33}=0.0594, \omega_{34}=0.0890, \omega_{35}=0.0118, \omega_{41}=0.0676$, $\omega_{42}=0.0271, \omega_{43}=0.0161, \omega_{44}=0.0113, \omega_{45}=0.0271, \omega_{46}=0.0161, \omega_{51}=0.0145 \omega_{52}=0.0579, \omega_{53}=0.0290, \omega_{54}=0.0058$.

The value in the table 1 into the equation, the ratio can be obtained between the actual detection value and the optimal detection value.

$$
\frac{W_{\text {actual }}}{W_{\text {optimal }}}=\left|\sum_{i} \sum_{j} \omega_{i j} f_{i j \text { (actual) }}\right| /\left|\sum_{i} \sum_{j} \omega_{i j} f_{i j \text { (optinal) }}\right|=\frac{6.4829}{7.9905}=81.17 \%
$$

It is up to 81.17 percent of the ideally, which reflect its detection ability. Cammanders Can make use of this result to determine this plan whether meets requirement.

\section{Summary}

The index system establishment is the premise of evaluation. Three levels of index evaluation system is given in the construction principles. The improved AHP is easy to get the largest eigenvalue 
and its corresponding eigenvectors and avoids the amount of calculation. The analysis of the example indicates that the improved AHP is good at the detection evaluation of radar network.

\section{Reference}

[1] L.Xiang, J.J.Ding, J.J.Lv. A study on dynamic evaluation of anticomplex jamming effect of radar netted system. Modern radar, Vol.32(2010), p.22-24. (in chinese)

[2] H.T. Zhang. Research on multi-band radar networking detecting and tracking of maneuvering stealthy target.Modern electronics echnique, Vol.37(2014), p.46-49. (in chinese)

[3] J. Tong , Z. S. LI,Q. Liu, et al. Modeling and simulation of radar networking of maritime maneuverable formation against stealth aircraft. Command control \&simulation, Vol. 35(2013), p.31-37. (in chinese)

[4] J.H. Li, W.Qin. Research on technology of radar survivability to anti-radiation missile. Modern navigation. Vol.4(2013),p.129-133.(in chinese)

[5] L.Zhao, P.X. Dong, T.Y. Fei, et al. Approach to Estimation of low altitude detection ability of radar network. Modern radar, Vol.30(2008), p.21-23. (in chinese)

[6] Y.Cao, G. Chen, W.G.Zhang, Z.M.Zhuo. A study on detection capability of radar seeker to low slow small target. Modern radar, Vol.34(2012), p.18-23. (in chinese)

[7] L.P. Yang, J.J Xiong. Radar netting detection capability evaluation based on SEA.Modern Defence Technology, Vol.39(2011), p.29-32. (in chinese)

[8] L.P. Yang, J.J Xiong. Evaluation index system of radar networking detection capabilities. Journal of air force radar academy, Vol. 25(2011), p.268-271. (in chinese)

[9] D.C. Huang, L.Xu. Proportion criteria and method for building comparison matrices in the analytic hierarchy process. Control and decision, Vol.17(2002), p.484-486. (in chinese)

[10] X.M.Wang, Q.Kang, J.C.Qin, et al. Application of AHP extenics model to safety evaluation of rock slope stability. Journal of central south university (science and technology), Vol. 44(2013), p.2455-2462. (in chinese) 